CAHIERS DE

NARRATOLOGIE
Cahiers de Narratologie

Analyse et théorie narratives

$19 \mid 2010$

Images composites, arts pluriels

\title{
Remise à zéro du langage : l'œuvre hybride de Ketty La Rocca (1938-1976)
}

\section{Cathy Margaillan}

\section{OpenEdition}

Journals

Édition électronique

URL : http://journals.openedition.org/narratologie/6197

DOI : 10.4000/narratologie.6197

ISSN : 1765-307X

Éditeur

LIRCES

Référence électronique

Cathy Margaillan, «Remise à zéro du langage : I'œuvre hybride de Ketty La Rocca (1938-1976) », Cahiers de Narratologie [En ligne], 19 | 2010, mis en ligne le 22 décembre 2010, consulté le 01 mai 2019. URL : http://journals.openedition.org/narratologie/6197; DOI : 10.4000/narratologie.6197

Ce document a été généré automatiquement le 1 mai 2019.

\section{(c) (i) (9)}

Cahiers de Narratologie - Analyse et théorie narratives est mis à disposition selon les termes de la licence Creative Commons Attribution - Pas d'Utilisation Commerciale - Pas de Modification 4.0 International. 


\title{
Remise à zéro du langage : l'œuvre hybride de Ketty La Rocca (1938-1976)
}

\author{
Cathy Margaillan
}

1 Dans les années soixante, soixante-dix, Florence apparaît comme le centre de la Néoavant-garde italienne ${ }^{1}$ où se développent des groupes de Poésie Visuelle et de Poésie Concrète tels que " Gruppo 63 » plus fidèle à une ligne littéraire ou "Gruppo 70 ». Ces groupes d'expérimentations littéraires et artistiques se créent autour de personnalités telles que E. Micini, U. Eco, L. Pignotti. C'est dans ce milieu qu'a évolué artistiquement et intellectuellement Ketty La Rocca qui est née à La Spezia mais qui a vécu à Florence à partir de 1949. Cette artiste peu connue et dont les œuvres ont souvent été oubliées, a joué un rôle essentiel dans la production artistique de ces années-là en adhérant au «Gruppo 70 », en poursuivant le discours subversif des avant-gardes historiques, en menant un discours critique par rapport au langage et aux médias alors en pleine évolution. En effet son adhésion au «Gruppo 70 » qui, comme groupe de Poésie visuelle entreprend une dénonciation de la subversion du langage des médias de communication et met en place une véritable "guérilla sémiotique », constitue une étape essentielle dans sa formation intellectuelle. Dans ce groupe, elle noue des liens avec les principaux représentants de la Poésie visuelle de l'époque notamment avec Eugenio Micini, Lamberti Pignotti, Mirella Bentivoglio, Lucia Marcucci. En 1963, elle participe au colloque « Arte e communicazione » organisé à Florence par ce même groupe. Ces contacts et ces activités avec le groupe lui permettent de construire le substrat nécessaire à sa réflexion sur le langage et surtout « d'apprendre l'utilisation des pratiques de contagion inter médiales parmi lesquelles on compte le collage et les activités liées au groupe ${ }^{2}$ ». Cependant même à l'intérieur du « Gruppo 70 », elle s'est toujours caractérisée par l'originalité de ses choix artistiques et de ses expérimentations.

2 Sa recherche sur le langage se veut personnelle, autonome et elle s'avère fortement corrélée à sa quête d'identité, à sa connaissance de soi dans son rapport au monde et aux autres. D'ailleurs la maladie qui l'a emportée à 38 ans, l'a très certainement conduite à 
ressentir de manière plus intense la nécessité d'un langage authentique qui lui permette de communiquer véritablement au plus vite. Sa réflexion sur le langage l'a amenée à explorer différents médias et à utiliser des supports très variés qui vont du collage à la photographie, en passant par les installations, les performances et à l'utilisation de la vidéo et de la télévision. Dans sa recherche, elle n'a pas hésité à tester les limites de ces différents moyens de communication, à les associer, à les juxtaposer, à réaliser des œuvres hybrides poly-matérielles et interdisciplinaires. Ces choix l'ont ainsi rapprochée des expériences de la pop art, du body art ou de la Narrative art mais sa diversité et son originalité demeurent intactes et ses concepts, son rapport au monde s'avèrent uniques. L'interaction entre les différents codes verbaux et visuels, l'utilisation de matériaux variés s'inscrivent dans sa recherche d'un langage authentique lui permettant de traduire le plus fidèlement possible, sans brouillage de la communication, sa conception du monde et son identité.

3 Pour atteindre cet objectif, son parcours intellectuel et artistique s'articule autour de deux moments fondamentaux qui vont correspondre aux deux premières parties de notre analyse. Dans un premier temps, elle part d'un constat: le langage de manière générale est corrompu par l'utilisation abusive, déviée qu'en ont fait les médias et la société de consommation; il n'est donc plus en mesure d'accomplir sa fonction première qui est la communication et surtout il ne permet plus à l'artiste d'exprimer librement son identité et sa conception du monde. Bref, elle dénonce la subversion du langage en utilisant le langage même des médias. Elle montre les limites et les problèmes que rencontre le langage et elle finit par conclure à son non-sens car il est devenu trop conventionnel. Sa réflexion et son expérimentation la conduisent à expérimenter le degré zéro des formes d'expression. Cette première phase d'expérimentation se situe autour des années 1963-1967 environ c'est-à-dire au moment où elle adhère au « Gruppo 70 ».

4 A partir de là, elle ressent le besoin d'entreprendre une reconstruction, de mettre en place un nouveau langage capable de traduire sa quête d'identité et son rapport au monde de manière efficace et authentique. Il s'agit de la deuxième phase qui se développe autour des années 1967-1976. Quand elle s'engage dans la reconstruction, elle emprunte les voies les plus diverses et elle associe, juxtapose, croise les codes verbaux, visuels, gestuels en utilisant des formes d'expressions nouvelles comme les Craniologie ou bien en se réappropriant les images par la calligraphie ou encore en faisant appel à la communication non verbale c'est-à-dire à celle gestuelle.

5 Cette réflexion sur le langage se caractérise par un double enjeu: d'une part, elle la conduit à s'interroger sur son identité de femme et d'artiste; d'autre part, ses choix artistiques c'est-à-dire les déconstructions, les recompositions, l'utilisation de matériaux différents, contribuent à redéfinir le concept d'œuvre d'art. En effet, les créations hybrides de Ketty la Rocca, ses performances et ses installations élargissent les frontières de l'œuvre d'art et elles transforment ainsi le rapport qui existe entre l'artiste et le récepteur. C'est pour cette raison que, comme nous le verrons dans une troisième partie, la coopération du récepteur devient essentielle dans la réalisation même de la création. A travers la réalisation de ses performances et de ses installations en particulier, elle insère l'art dans la vie et vice-versa au point que le récepteur devient partie intégrante de l'œuvre.

6 L'adhésion de Ketty La Rocca au « Gruppo 70 », sa participation au colloque sur « l'art et la communication » en 1963 et ses lectures de U. Eco, notamment La struttura assente, de Barthes, de Mac Luhan, de Levi-Strauss lui fournissent les bases de sa réflexion sur le 
langage et la communication. Elle s'engage dans des expérimentations qui la rapprochent d'autres femmes artistes telles que Lucia Marcucci, Mirella Bentivoglio ou Irma Blank. Ces expérimentations comprennent trois phases essentielles alliant des techniques et des matériaux différents. Tout d'abord elle entreprend une déconstruction du langage en faisant interagir les codes verbaux et visuels. Ensuite elle vide les mots de leur sens et leur confère une seule valeur iconique et enfin elle conclut au non-sens du langage en soulignant combien les mots ne sont que des simulacres.

7 Ketty La Rocca part d'un constat qu'elle partage avec les autres membres du «Gruppo 70 » à savoir que le langage traditionnel est devenu conventionnel, il a tellement été corrompu par la culture et les mass-médias qu'il a perdu sa signification et qu'il ne remplit plus sa fonction de communication voire même il tend à brouiller le message entre les hommes et à créer l'ambiguïté. Elle souligne cette carence en faisant interagir les images et les mots, les codes visuels et les codes verbaux à travers ses collages. Cinq collages se distinguent plus particulièrement car ils permettent de mettre en évidence les caractéristiques principales de cette phase de subversion du langage : il s'agit de Sana come il pane quotidiano (1964-65), Vergine (1964-65), Sono felice (1965), Qualcosa di vecchio (1964-65), Bianco Napalm (deux versions: une en 1966 et l'autre en 1967) ${ }^{3}$. Dans ses collages, Ketty La Rocca utilise des images, des mots ou des expressions découpées dans les journaux, dans les magazines illustrés qu'elle juxtapose, associe, mélange afin de montrer le brouillage de la communication. En effet dans ses collages, Ketty La Rocca dévie les images et les mots de leur contexte habituel et elle les fait agir de manière autonome. C'est en utilisant le langage, les moyens et les formes d'expression du langage des médias que notre artiste en dénonce la subversion. C'est donc de l'intérieur que Ketty La Rocca déconstruit le langage. Les mots ne sont pas l'illustration de l'image publicitaire proposée. En dé-contextualisant et en réorganisant les messages verbaux et iconiques, elle finit par obtenir le message contraire car les mots et les images sorties de leur contexte renvoient à des conventions et à des stéréotypes. Au niveau des habitudes de réception de l'œuvre, le bouleversement est total: les mots sortent de la linéarité de la page, ils sont souvent écrits en diagonale ou en superposition avec l'image de sorte qu'un véritable dialogue s'instaure entre l'image et les mots et le spectateur ressent l'effet de surprise. De plus le message ne prend sa véritable signification que si le lecteur/ spectateur fait interagir les deux codes de nature différente. Ces compositions souvent en noir et blanc se définissent par leur aspect essentiel et elles impliquent non seulement la dénonciation du langage, la critique de la société et plus particulièrement des stéréotypes qu'elle véhicule sur la femme mais aussi un engagement politique car elles constituent une prise de position souvent anticléricale et anticolonialiste. En effet à travers les quatre premiers collages cités, la réflexion sur le langage de Ketty La Rocca apparaît étroitement liée à sa conception de la réalité. Ainsi dans Sana come il pane quotidiano (1964-65), Vergine (1964-65), Sono felice (1965), Qualcosa di vecchio (1964-65), en utilisant un langage fondé sur la conflictualité, elle dénonce l'image de la femme véhiculée par la société de consommation se rapprochant ainsi des dénonciations féministes. Cependant elle n'a jamais adhéré aux mouvements féministes car son objectif est personnel et elle recherche un langage en mesure d'exprimer son identité et lui permettant de renouer un dialogue avec les autres. Son premier collage, réalisé en 1965 Sana come il pane quotidiano représente le corps d'une femme belle et saine telle que l'on pouvait la voir dans les magazines de l'époque illustrant la pureté du corps féminin. Dans la partie inférieure, un médaillon représentant des enfants pauvres du tiers monde se superpose au corps de la femme. En haut à droite en diagonale, des mots en lettres typographiques découpés dans 
les journaux reprennent les termes de la prière " come il pane quotidiano ${ }^{4}$ ». Ces termes de la prière sont associés au terme "sana ${ }^{5}$ ». Les interprétations sont multiples mais toutes dénoncent la représentation de la femme comme objet sexuel ou de désir. Pour L.

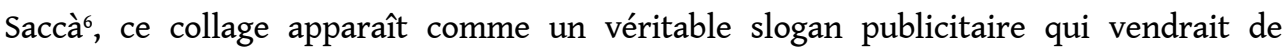
manière provocatrice le corps de la femme saine comme on mettrait en vente le pain. En revanche E. Del Becaro ${ }^{7}$ met en évidence une réflexion plus profonde et plus marquée par l'éducation catholique de notre artiste : le pain de la prière, c'est également le moment de la transsubstantiation, ici c'est le corps de la femme qui devient nutriment des fidèles ce qui est un péché; elle souligne ainsi l'hypocrisie de l'Eglise. Ce collage se fonde sur la conflictualité entre le visuel et le verbal : aux formes rondes de l'image et du corps de la femme s'oppose l'écriture typographique; cependant les mots et les images se répartissent l'espace de manière équitable. Cette technique permet de dénoncer la représentation de la femme comme objet sexuel et elle est reprise dans les collages qui ont suivis. Dans Vergine, dans Sono Felice, et dans Qualcosa di vecchio, elle continue de se pencher sur la condition de la femme dans la société de son époque, c'est-à-dire dans une société de type patriarcal qui définit la femme avant tout comme une épouse et une mère. Dans Vergine, le corps de la femme est considéré comme n'importe quel produit de consommation. Dans Sono Felice, avec sarcasme, Ketty La Rocca montre que les critères de beauté et de jeunesse sont imposés à la femme par les clichés publicitaires et elle souligne que le modèle de la femme présenté est celui de la femme au foyer qui doit se montrer heureuse. Le modèle qu'ici notre artiste conteste, est celui encouragé par les institutions et les médias. De la même manière, dans Qualcosa di vecchio, comme l'indique le titre, elle insiste sur le fait que rien n'a vraiment changé dans la société. La femmes n'a acquis finalement qu'un semblant de libertés car la société continue de la considérer comme un objet. Elle a un discours anticipateur en affirmant que la totale liberté sexuelle accordée à la femme ne va pas lui permettre d'être plus libre.

8 Sa recherche d'un langage authentique a conduit Ketty La Rocca à s'approcher des dénonciations féministes mais également à s'engager politiquement contre le colonialisme notamment à travers deux collages particulièrement significatifs : Bianco Napalm (1966) et Le scimmie impareranno a parlare? (1964-65) ${ }^{8}$. Dans les deux cas, la technique choisie est celle de la composition conflictuelle qui lui permet de synthétiser les conflits existants à travers l'opposition image vs verbe. Dans Le scimmie impareranno a parlare? qui est un collage en couleur, c'est la famille blanche baignant dans le luxe qui est opposée à la famille noire pauvre. Dans Bianco Napalm, (www.trans.univ-paris3.fr/ spip.php?article93) elle se concentre sur l'essentialité du message et l'aspect synthétique en privilégiant l'aspect iconique. Dans ce collage le blanc domine tant au niveau iconique que verbal. Il n'y a pas de fond, tout est blanc, et même du prêtre il ne reste que le collet blanc. Généralement le terme "Bianco » entraîne une série de sensations différentes qui renvoient à la pureté, au silence, à l'absence; ici il revêt un aspect négatif puisqu'il est associé au Napalm, symbole de la guerre du Vietnam et le collet blanc du prêtre symbolise le silence de l'Eglise face au massacre. La dénonciation de l'hypocrisie de l'Eglise est confirmée par l'antagonisme entre les différentes formes de pouvoir qui, dans le collage, sont représentées par les trois catégories de pouvoir de l'Antiquité Oratores, bellatores et laboratores à travers les figures du prêtre, du militaire et de la mère. La représentation de ces trois catégories contribue à souligner qu'au cours des siècles, rien n'a changé quant au pouvoir. Au niveau de la technique, dans ce collage, une légère asymétrie dans l'association des images et des mots se dessine; les termes «Bianco Napalm » occupent une plus grande partie de l'espace reléguant sur le côté droit l'image de la femme, de 
l'enfant et du militaire. Ketty La Rocca modifie sa technique de collage, elle devient plus précise dans la mise en place des éléments, et elle s'interroge sur la taille du collage. Ainsi la deuxième version de ce collage qui est réalisée sur un format plus grand, annonce le passage de Ketty La Rocca du collage aux arts figuratifs.

9 Après avoir montré les limites du langage et en avoir dénoncé la corruption, dans une deuxième phase, elle va poursuivre sa réflexion en montrant le non-sens du langage et plus particulièrement en insistant sur la matérialité des lettres et des mots. Dans cette deuxième phase, pour Ketty La Rocca le mot devient un signifiant qui n'est utilisé que pour sa valeur iconique : le mot perd toute sa signification, il devient un objet. Le collage Io sono Peter (1964-65) ${ }^{9}$ marque un tournant dans la recherche de l'artiste qui prend conscience que « il solo accostamento e dissidio tra parola e immagini sono insufficienti ${ }^{10}$ ». Il annonce de nouvelles voies à explorer et les changements de supports à venir. En effet, l'écriture n'est plus typographique mais manuscrite, les lettres sont utilisées comme des objets et non pour être associées et ainsi créer des mots ayant du sens. Dans ce collage, plusieurs prêtres traversent la lettre « 0 » insistant ainsi sur l'aspect matériel et physique de la lettre. Ketty La Rocca dénonce ainsi le non-sens des affirmations de l'Eglise. Dans ce collage en conférant une importance notable à la valeur iconique de la lettre, elle anticipe la réalisation des lettres géantes ${ }^{11}$ tridimensionnelles en PVC noir qu'elle installera dans les jardins publics. De plus, avec ce collage, le «Moi » fait son irruption; par la suite le sujet deviendra de plus en plus présent et se verra associé à "l'autre » sous la forme des termes «you-you ». Cette présence toujours plus imposante du sujet souligne le passage de Ketty La Rocca de la sphère publique à la sphère privée, personnelle.

10 Après ce collage Io sono Peter, elle passe à l'utilisation des panneaux de signalisation ( www.mart.tn.it/gallery.jsp?ID_LINK=96\&area=42...) existants qu'elle transforme ou bien des panneaux de signalisation qu'elle invente totalement en utilisant des phrases, des mots conventionnels. Les mots sont utilisés dans des œuvres pour leur valeur iconique complètement vidés de sens, et utilisés seuls en dehors de tout contexte : l'objectif étant de souligner le non-sens du langage. Elle choisit des mots ou des morceaux de phrases écrits en lettres typographiques ou manuscrites qu'elle isole de tout contexte montrant une volonté d'épuration. Parfois même elle n'utilise que la ponctuation. Le signifié est généralement celui des conventions alors qu'elle recompose le signifiant laissant une grande ouverture à l'interprétation. Cette réappropriation du langage chez Ketty La Rocca correspond à une recherche de soi. Il s'agit tout particulièrement des œuvres tels que In pricipium erat (1967), Verbum (1967) (www.galerieimtaxispalais.at/ archiv_1999-2008/ausstellungen/almeida_larocca/larocca_rundgang2.htm) ou encore Era quasi buio... (1968-69) qui se présentent comme des phrases seules, hors contexte à compléter laissant la plus grande ouverture d'interprétations possibles. Elle procède de la même manière dans le cadre des performances comme Approdo ${ }^{12}$ en 1967 où elle tente de démonter les engrenages de la communication afin de pouvoir créer un dialogue plus profond avec le récepteur. Dans cette performance, tout comme dans Io sono Peter, le «moi » fait irruption dans son rapport à l'autre et au monde. Avec les panneaux de signalisation, elle montre que les mots se sont vidés de leur sens ; le même discours peut s'appliquer aux images publicitaires, ou aux affiches des films cultes hollywoodiens ou aux photographies d'œuvres d'art qui appartiennent à la culture de masse et qui ont été tellement utilisées dans des contextes si différents qu'elles finissent par ne plus rien signifier. Ainsi elle les décompose et elle se les réapproprie en ne laissant que le contour 
dessiné dans une calligraphie illisible : il s'agit des Riduzioni ${ }^{13}$ notamment celles intitulées David (1973) (www.arcadja.com), Via col vento (1975) (www.galerieimtaxispalais.at/ archiv_1999-2008/ausstellungen/almeida_larocca/larocca_rundgang2.htm ), Fidel Castro (1974).

11 Elle dénonce le langage des conventions qui trahit la signification des mots et finit par vider de leur sens les mots et les images qui finissent par ne plus rien signifier. Dans deux de ces créations tout particulièrement Dal momento in cui (1970) et dans Firenze Museo del Bargello (1972) ${ }^{14}$, Ketty La Rocca va plus loin en montrant que les mots ont tellement été utilisés qu'ils sont réduits à n'être que des simulacres vides qui ne signifient rien d'autre qu'eux-mêmes : ils constituent un vase clos rendant impossible la communication. Ainsi dans ces deux œuvres, même si la syntaxe est correcte, il ne s'agit que d'une juxtaposition de mots qui ne prennent pas de sens entre eux. Ils reflètent un rejet du dialogue et une fermeture des individus les uns par rapport aux autres. Ou encore par la répétition des mêmes mots, des mêmes morceaux de phrases ou d'expression, l'artiste souligne la perte de sens du langage qui devient artificiel. Elle nourrit ainsi une méfiance à l'égard de la communication verbale car elle a pris conscience des limites des mots dans la recherche d'une communication authentique.

Sa réflexion et son expérimentation l'ont conduite à un degré zéro des formes d'expression. A partir de là, il lui faut s'engager dans la reconstruction d'un langage authentique qui lui permette d'affirmer son identité, d'exprimer sa conception du monde et son rapport aux autres. Pour y parvenir, elle va emprunter les voies les plus diverses qui vont des recherches liées à la communication non verbale, à la gestualité, aux performances, aux installations, à la télévision, aux Riduzioni, (www.essogallery.com/ Ketty\%20La\%20Rocca/KLR_Images.html ) et aux Craniologie ${ }^{15}$ ( www.sguardodonna.wordpress.com). Elle désire un langage capable de faire dialoguer son intériorité avec l'extérieur, avec le monde et les autres et ce désir se fait ressentir de manière encore plus pressante à partir du moment où dans les années soixante dix, elle découvre la présence de sa tumeur. Ce besoin omniprésent d'entrer en contact avec les autres dans cette deuxième phase de son parcours entre 1969 et 1976, année de son décès, ressort de la présence des mots "you-you " qui demeurent lisibles même dans les calligraphies. Trois phases peuvent être distinguées dans son parcours de réappropriation d'un langage authentique. Tout d'abord, sa recherche d'universalité du langage la conduit à accorder une importance particulière à l'écriture manuscrite et aux gestes. Elle se tourne vers la communication non-verbale et vers la gestualité à travers une série de photographies de ses mains dans Le mie parole $e$ tu? (1971-72) (www.galerieimtaxispalais.at/archiv_1999-2008/ausstellungen/almeida_larocca/ larocca_progindex_ital.htm) et à travers la vidéo Appendice per una supplica ${ }^{16}$ (1972) ( www.galerieimtaxispalais.at). Ces créations renouent avec les dénonciations féministes du rapport entre homme et femme qui étaient déjà présentes dans les premiers collages qu'elle a réalisés. Ainsi, dans certaines photographies, elle dénonce l'emprise de la main de l'homme sur celle de la femme mais en même temps, elle insiste sur la possibilité d'un rapport de communication plus direct, plus efficace entre les êtres grâce aux gestes. Toutefois, elle n'abandonne jamais les mots ou plus précisément la graphie. En effet dans certaines photographies des gestes, les mots you-you sont visibles et la calligraphie reste présente en interaction avec la gestualité. Le choix de la gestualité montre qu'elle tente de faire appel à tous les sens pour parvenir à une communication plus authentique et plus immédiate. 

l'écriture manuscrite en créant un livre-objet fait main ${ }^{17}$. Cela est confirmé dans les Riduzioni, www.eramoderna.it/.../women_from_60_s_\&_70_s.htm) où les textes calligraphiés illisibles renvoient à l'origine non-verbale du texte. La calligraphie illisible devient contour de l'image afin de signaler la séparation entre l'intérieur et l'extérieur et la nécessité de lier les deux pour affirmer son identité et pour pouvoir communiquer. Ici aussi le mot you demeure toujours lisible, il sert de lien entre le « moi » et les autres.

Elle persiste à développer ce lien entre intérieur et extérieur, dans une troisième phase de son parcours à savoir à travers ses Craniologi $e^{18}$, qui se présentent comme de véritables Vanitas modernes où l'image médicale devient image artistique et où l'art et la vie ne font qu'un. Dans ces œuvres, l'image, le geste et le texte ou la graphie se superposent pour traduire au mieux l'identité de l'artiste et permettre à l'extérieur de rentrer en interaction avec l'intérieur de l'être. Les Craniologie montrent qu'elle a fini par mettre en place son propre système de communication avec des codes qui lui sont propres et qui expriment véritablement son identité ; pour elle, «L'union de son être tout entier à son travail et l'adéquation intime et substantielle avec les instruments de sa communication sont complets. L'art et la vie pour elle coïncident totalement et ici elle choisit de faire don de soi à travers les empreintes les plus tragiques de son existence ${ }^{19}$ ». Le problème se situe au niveau de la réception car le lecteur/spectateur a besoin d'accéder à ces codes pour comprendre son message. C'est pourquoi dans ses expositions, les écrits constituent un commentaire de ses œuvres d'arts visuels. choix artistiques, le choix de ses matériaux, la juxtaposition et l'interaction de codes de nature différents, Ketty La Rocca a élargi les frontières de l'œuvre d'art entre langage verbal et les arts visuels et elle a ainsi poursuivi le discours amorcé par les avant-gardes historiques. En tentant de trouver des solutions nouvelles pour une communication plus authentique et plus fiable, Ketty La Rocca a instauré un nouveau rapport avec le récepteur qui a conduit à une redéfinition du concept d'œuvre d'art. Dans les différentes expérimentations -du collage, aux Riduzioni, aux Craniologie, en passant par les performances, les installations, la vidéo et les photographies des gestes- dans l'ensemble de ses œuvres hybrides, Ketty La Rocca réclame la coopération totale du récepteur; le contact avec le récepteur, son interactivité s'avèrent essentielles. Sans la collaboration du public, l'œuvre n'existe pas. Ce rapport avec le récepteur évolue au fur et à mesure de sa réflexion sur le langage. Dans la première phase de son expérimentation, les collages passent par la déstabilisation du récepteur qui voit des codes différents interagir alors que d'habitude ils fonctionnent séparément; ainsi « l'horizon d'attente du spectateur est invalidé puisqu'il ne parvient plus à reconnaître les conventions propres à l'œuvre et d'autant moins encore les transgressions ${ }^{20} »$. Toutefois c'est l'interprétation du récepteur qui souligne le contraste entre l'image et les mots et qui confère du sens au collage. L'effet de surprise est essentiel pour amener un public élargi à réfléchir sur le langage et la société dans laquelle il vit. D'ailleurs Ketty La Rocca précise « il faut garder à l'esprit que pour sensibiliser un public de non expert il est nécessaire d'utiliser l'effet de surprise ${ }^{21}$ ».

16 Par la suite avec les panneaux de signalisations où l'ellipse prévaut, elle oblige le récepteur à compléter la phrase. Ainsi Ketty La Rocca offre à l'observateur « des œuvres achevées mais partielles ${ }^{22}$ » car « la sienne [...] est une intention constante de coopération avec l'autre, dans un rapport spéculaire et dialogique qu'elle poursuivra avec d'autres médias tels que l'installation et la performance ${ }^{23}$ ». Cette recherche de coopération avec 
l'autre est mise en évidence par le leitmotiv manuscrit you-you que l'on retrouve dans les Riduzioni, et dans les Craniologie.

Ainsi des collages où le récepteur devait déchiffrer l'œuvre, on passe aux performances et aux installations où le récepteur devient partie intégrante de l'œuvre. Dans les performances de Ketty la Rocca, Approdo en 1967, Volantini sulla strada toujours en 1967, on assiste à une recherche de relation directe avec le spectateur par l'action dans l'espace urbain. Avec ses installations Specchi ${ }^{24}(1967)$ et Il punto di vista ${ }^{25}$ (1969), Ketty La Rocca s'intéresse aux dynamiques qui existent entre l'œuvre et le spectateur. Par définition, l'installation constitue un type de création artistique qui se penchent sur les relations et les interactions entre les choses et leur contexte.

Pour Ketty La Rocca l'installation devient « un dialogue continuel entre le récepteur et le dispositifi ${ }^{26}$ » et à chaque progression du récepteur à l'intérieur de l'installation, une réception nouvelle a lieu qui crée un nouvel évènement. De plus le spectateur perd ses points de repère conventionnels: dans l'installation Gli specchi, (www.undo.net/it/ magazines/1014743447) l'œuvre devient mobile et vivante et les miroirs suscitent la perte d'orientation du spectateur. Bref, des créations hybrides de Ketty la Rocca, il ressort que la collaboration du récepteur et son interactivité sont indispensables pour que l'œuvre d'art existe et qu'une communication authentique ait lieu.

Ketty La Rocca souvent définie "la diversa", a su mettre en place un système de communication personnel et original. C'est en interrogeant constamment les différents médias, en testant les différents supports, en menant une réflexion poussée sur la communication et les différentes formes de langage que l'artiste a atteint son objectif. Ses recherches l'insèrent dans le contexte artistique international de l'époque tout en lui ayant permis de construire son identité et son rapport au monde de manière très autonome. Ses œuvres hybrides reflètent sa manière de concevoir le monde mais en même temps posent les problèmes fondamentaux concernant l'œuvre d'art à savoir sa définition et sa réception.

\section{BIBLIOGRAPHIE}

A. Bonito Oliva (dir.), Le presenze alfabetiche e lo spazio parlato, Comune di Modena, Istituti Culturali, 1970.

E. Belsito (dir.), Ketty La Rocca, j, La Spezia, Fondazione Cassa si Risparmio della La Spezia, 1999.

L. Saccà (dir.), Omaggio a Ketty La Rocca, Comune di Monsummano Terme, Pacini editore, 2001.

L. Saccà (dir.), Ketty La Rocca : i suoi scritti, Turin, Martano Editore, 2006.

E. Del Becaro, Intermedialità al femminile : l'opera di Ketty la Rocca, Milan, Electa Mondadori, 2008.

E. Biagini, «L'ossessione del linguaggio : le prime opere di Ketty La Rocca », Italian Culture, 19, 2001, p. 111-126.

J.R. Kirshner, « You and I the art of Ketty La Rocca », Art forum international, 7, 1993, p. 82-84. 


\section{ANNEXES}

\section{Sites internet}

http://germangalleries.com/KienzleGmeiner/LaRocca.html

http://www.frugalfun.com/essogallery.html

http://www.essogallery.com/laRocca.html

http://www.galerieimtaxispalais.at/ausstellungen/almeida_larocca/

larocca_progindex_ital.html

http://www.kienzle-gmeiner.de

http://www.galleriacarini.com/carini/mostre/8-15/kettylarocca-precedenti-2.html

\section{NOTES}

1. Même si Florence apparaît comme le berceau du phénomène, d'autres zones d'Italie voient se développer des expérimentations analogues liées à la poésie visuelle: à Gênes, il y a le groupe dirigé par Martino et Ana Oberto, à Rome, la présence de Mirella Bentivoglio est significative pour la Poésie visuelle. Cf. E. Del Becaro, Intermedialità al femminile : l'opera di Ketty La Rocca, Milan, Electa Mondadori, 2008, p. 31.

2. E. Del Becaro, Intermedialità al femminile : l'opera di Ketty La Rocca, cit., p. 24. " apprende l'uso di pratiche di contaminazione intermediali, tra cui il collage e le attività performative ».

3. E. Del Becaro, Intermedialità al femminile : l'opera di Ketty La Rocca, cit., p. 211 et suivantes.

4. «Comme le pain quotidien ».

5. « saine".

6. L. Saccà (dir.), Omaggio a Ketty La Rocca, Comune di Monsummano Terme, Pacini editore, 2001, p. 30 .

7. E. Del Becaro, Intermedialità al femminile : l'opera di Ketty La Rocca, cit., p. 77.

8. L. Saccà (dir.), Omaggio a Ketty La Rocca, cit., p. 37, 38.

9. Ibid., p. 41.

10. E. Del Becaro, Intermedialità al femminile: l'opera di Ketty La Rocca, cit., p. 102. «Le seul rapprochement et la seule opposition entre mot et images s'avèrent insuffisants ".

11. L. Saccà (dir.), Omaggio a Ketty LaRocca, cit., p.61, Installazione con virgole, p.64, Installazione con J.

12. Approdo qui se situe entre l'installation et la performance. Les artistes ont substitué les panneaux de signalisations par des œuvres d'art sur l'autoroute au niveau de l'échangeur de Florence; autoroute qui a été fermée 15 minutes le temps de la performance. Parmi les œuvres présentes sur l'autoroute celles de Ketty La Rocca les panneaux de signalisations : Noi2, io e tu e le rose, Engagement, Mia6. in E. Del Becaro, Intermedialità al femminile : l'opera di Ketty La Rocca, cit., p. 110.

13. L. Saccà (dir.), Omaggio a Ketty La Rocca, cit., p. 130, 139, 163, 165.

14. Ibid., p. 75, 76, 77.

15. Ibid., p. 101 et suivantes. Les Craniologie : il s'agit de radiographies médicales du crâne de Ketty La Rocca qui souffre d'une tumeur. Elle pratique la calligraphie autour de son crâne sur la 
radiographie où elle superpose la photographie de son poing à l'intérieur de la radiographie de son crâne.

16. L. Saccà (dir.), Omaggio a Ketty La Rocca, cit., p. 88 et suivantes.

17. Ibid., p. 87-88.

18. Ibid., p. 101-109.

19. E. Del Becaro, Intermedialità al femminile : l'opera di Ketty La Rocca, cit., p. 204. « l'unione di tutta sè stessa al proprio lavoro e l'aderenza intima e sostanziale con gli strumenti della sua communicazione artistica sono totali. L'arte e la vita sono per lei veramente identità e qui scegli di far dono di sè mediante le più tragiche impronte del suo vissuto ».

20. Ibid., p.122. «si invalida così l'orizzonte d'attesa poiché lo spettatore non riesce a riconoscere nessuna convenzione nell'opera né tantomeno le sue trasgressioni ».

21. L. Saccà, Ketty La Rocca : $i$ suoi scritti, cit., p. 113. «Occore tener presente che per sensibilizzare un pubblico di non esperti è necessario passare attraverso la fase della sorpresa ».

22. E. Del Becaro, Intermedialità al femminile : l'opera di Ketty La Rocca, cit., p. 107. « opere finite ma parziali ».

23. Ibidem. " la sua è [...] un'intenzione di cooperazione con l'altro, in un rapporto speculare e dialogico che perseguire anche con altri media, quali l'installazione e la performance» «la sienne [...] est une intention constante de coopération avec l'autre, dans un rapport spéculaire et dialogique qu'elle poursuivra avec d'autres médias tels que l'installation et la performance ».

24. L. Saccà (dir.), Omaggio a Ketty La Rocca, cit., p. 62-63.

25. L. Saccà (dir.), Omaggio a Ketty La Rocca, cit., p. 66-67.

26. E. Del Becaro, Intermedialità al femminile : l'opera di Ketty La Rocca, cit., p. 120. « un dialogo continuo tra il fruitore e il dispositivo ».

\section{RÉSUMÉS}

Artiste italienne, née à La Spezia, Ketty La Rocca a évolué, au cours des années 70, en adhérant, dans un premier temps, au groupe 70 de « Poesia Visiva » et elle a ensuite poursuivi sa recherche de manière autonome. Il s'agit d'une artiste qui, à travers ses œuvres poly-matérielles et interdisciplinaires, développe une réflexion sur le langage. Elle entreprend l'exploration des différents moyens de communication en juxtaposant, croisant, mélangeant, les différents matériaux (collages de mots, de photographies issus des médias et de la société de consommation). Ses expérimentations la conduisent à s'interroger d'abord sur le rapport entre les mots et l'image puis uniquement sur les mots et elle finit par conclure au non sens du langage ; ce dernier s'avère faux et corrompu, il ne permet plus d'accomplir sa fonction première qui est la communication entre les êtres humains et surtout il ne parvient plus à exprimer en toute authenticité et liberté l'identité de l'artiste. Sa réflexion et son expérimentation la conduisent à un degré zéro des formes d'expression. A partir de là, elle s'engage dans la reconstruction en empruntant les voies les plus diverses (recherches liés à la communication non verbale, la gestualité, les performances, la télévision, les installations, Riduzioni, Craniologie...). Cette remise à zéro du langage a lieu pour répondre à sa réflexion personnelle, sur son être, sur son rapport au monde et aux autres et plus particulièrement elle participe à la réappropriation de son identité. 
Ketty La Rocca è un'artista italiana nata a La Spezia e vissuta a Firenze negli anni settanta. Il suo percorso artistico e intellettuale è segnato dalla sua adesione al "Gruppo 70" di Poesia Visiva. Ma presto ha lasciato il gruppo per proseguire le proprie ricerche in modo autonomo. S'interroga particolarmente sul linguaggio e la sua funzione attraverso varie sperimentazioni e l'uso di materiali diversi. Esplora vari mezzi di comunicazione giustapponendo, mescolando, associando vari materiali (collages di parole, fotografie tratte di mass media e dalla società di consumo). Queste sperimentazioni la portano a riflettere sul rapporto tra parola e immagine poi unicamente sulle parole per concludere al non senso del linguaggio. Quest'ultimo appare falso e corrotto al punto da non compiere più la sua funzione principale vale a dire il comunicare tra gli esseri umani ; di più il linguaggio non consente più di esprimere in modo autonomo e autentico l'identità dell'artista. Giunge così al grado zero delle forme espressive. Da quel momento, Ketty La Rocca s'impegna nel ricostruire il linguaggio imboccando le vie più diverse e usando mezzi vari (ricerche legate alla comunicazione non verbale, la gestualità, le performance, televisione, installazioni, Riduzioni, Craniologie...). Questo suo azzerare il linguaggio le consente di rispondere alla riflessione personale che avvia sul proprio essere, sul proprio rapporto con il mondo e gli altri e soprattutto le permette di ri-appropriarsi la propria identità. Nel suo percorso artistico e intellettuale s'impegna al livello politico e femminista prendendo posizioni anticolonialiste e denunciando lo stereotipo della donna tramandato dalla società di consumo e dai mass media.

\section{INDEX}

Mots-clés : collages, féminisme, installations, langage, La Rocca (Ketty), Néo-avant-garde, œuvre d'art, performances, Poésie visuelle, réception

\section{AUTEUR}

\section{CATHY MARGAILLAN}

Université de Nice-Sophia Antipolis 